\title{
Time in relativistic and nonrelativistic quantum mechanics
}

\author{
Hrvoje Nikolić \\ Theoretical Physics Division, Rudjer Bošković Institute, \\ P.O.B. 180, HR-10002 Zagreb, Croatia \\ e-mail: hrvoje@thphys.irb.hr
}

October 3, 2018

\begin{abstract}
The kinematic time operator can be naturally defined in relativistic and nonrelativistic quantum mechanics (QM) by treating time on an equal footing with space. The spacetime-position operator acts in the Hilbert space of functions of space and time. Dynamics, however, makes eigenstates of the time operator unphysical. This poses a problem for the standard interpretation of $\mathrm{QM}$ and reinforces the role of alternative interpretations such as the Bohmian one. The Bohmian interpretation, despite of being nonlocal in accordance with the Bell theorem, is shown to be relativistic covariant.
\end{abstract}

PACS numbers: 03.65.Ta, 03.65.Pm

Keywords: Time; Quantum mechanics; Relativity; Bohmian interpretation

\section{Introduction}

The problem of time-operator in quantum mechanics (QM) is an old, still unsolved problem (see, e.g., 1, 2, 3] for reviews). In its most elementary form, the problem can be stated as an old observation [4] that, if the Hamiltonian $\hat{H}$ is bounded from below, there can be no self-adjoint time-operator $\hat{T}$ satisfying the canonical commutation relation

$$
[\hat{T}, \hat{H}]=-i \hbar .
$$

This is especially problematic for relativistic QM, because in a relativistic theory one expects that time should be treated on an equal footing with space. Guided by the idea that time should be treated on an equal footing with space, in this paper we propose a simple solution of that problem. Essentially, when acting on a wave function $\psi(\mathbf{x}, t)$, the time operator acts as a multiplication with the parameter $t$. To make it meaningful, the Hilbert space in which the physical operators act must be enlarged from the space 
of functions $f(\mathbf{x})$ of $\mathbf{x}$ to the space of functions $f(\mathbf{x}, t)$ of both $\mathbf{x}$ and $t$. The quantity $|\psi(\mathbf{x}, t)|^{2} d^{3} x d t$ is then naturally interpreted as the probability that the particle will be found in the (infinitesimal) spacetime volume $d^{3} x d t$.

Some forms of this idea have also been proposed in the context of proper-time quantization of relativistic particles [5], relativistic quantum measurements [6], and measurement events [2]. However, even though such a construction works perfectly well on the kinematic level (i.e., without using the wave equation of motion for $\psi(\mathbf{x}, t)$ ), the common and unsolved problem of all these previous attempts is the fact that it does not work on the dynamical level, essentially because eigenstates of the time operator are not solutions of the equation of motion. In this paper we propose a solution of that problem as well. Moreover, we discuss how several other foundational problems of QM are naturally solved within this approach. More specifically, the list of advantages of this approach includes the following:

- Space and time operators are defined in a relativistic covariant way.

- A relativistic covariant probabilistic interpretation of solutions of the Klein-Gordon equation is given.

- The standard interpretation of transition amplitudes in terms of transition probabilities per unit time is now better founded in basic axioms of QM.

- The fact that not all interpretations of QM are compatible with the fact that eigenstates of the time operator are unphysical provides a new guiding principle towards a resolution of notorious interpretational ambiguities related to the measurement problem in QM.

- In the Bohmian interpretation the dynamical absence of time-eigenstates does not represent a fundamental problem.

- Even though the Bohmian interpretation is not local, it is shown to be relativistic covariant.

In the rest of the paper we explain these properties in more detail. From now on, we use natural units $\hbar=c=1$ and the relativistic metric signature $(+,-,-,-)$.

\section{Kinematics}

Let us start with a purely kinematic analysis. We introduce the standard relativistic notation $x=\left\{x^{\mu}\right\}, \mu=0,1,2,3$, where $x^{0} \equiv t$ and $\left\{x^{1}, x^{2}, x^{3}\right\} \equiv \mathbf{x}$. The kinematic 4-momentum operator is

$$
\hat{p}_{\nu}=i \partial_{\nu}
$$

which satisfies the covariant canonical commutation relations

$$
\left[x^{\mu}, \hat{p}^{\nu}\right]=-i g^{\mu \nu}
$$

The time operator is simply the $x^{0}$ component of $x^{\mu}$. Similarly, the space-position operator is the space-component $\mathbf{x}$ of $x^{\mu}$. Thus, unlike the Newton-Wigner position operator [7], 
our position operator is relativistic covariant by being a space-component of a Lorentz 4 -vector. The time-component of (2) is the kinematic energy operator

$$
\hat{E}=i \frac{\partial}{\partial t}
$$

which may serve as an energy operator even in the nonrelativistic case. Thus, instead of the inconsistent relation (1), Eq. (3) leads to a consistent one

$$
[t, \hat{E}]=-i
$$

Unlike the dynamical Hamiltonian operator $\hat{H}$, the kinematic energy operator $\hat{E}$ is not bounded from below. The operators (2) act on the space of functions $f(x)$ of $x$. The natural scalar product on this space is $\left\langle\psi \mid \psi^{\prime}\right\rangle=\int d^{4} x \psi^{*}(x) \psi^{\prime}(x)$. In particular, if $\psi$ is normalized such that $\langle\psi \mid \psi\rangle=1$, then the quantity

$$
d P=|\psi(x)|^{2} d^{4} x
$$

is naturally interpreted as the probability of finding the particle in the (infinitesimal) 4 -volume $d^{4} x$.

At first sight, (6) may seem to be incompatible with the usual probabilistic interpretation in 3-space

$$
d P_{(3)}=|\psi(\mathbf{x}, t)|^{2} d^{3} x .
$$

Nevertheless, (6) is compatible with (77). If (6) is the fundamental a priori probability, then (7) is naturally interpreted as the conditional probability corresponding to the case in which one knows that the particle is detected at time $t$. More precisely, $\psi$ in (6) and (7) have different normalizations, so a more precise form of (7) is

$$
d P_{(3)}=\frac{|\psi(\mathbf{x}, t)|^{2} d^{3} x}{N_{t}},
$$

where

$$
N_{t}=\int d^{3} x|\psi(\mathbf{x}, t)|^{2}
$$

is the normalization factor. If $\psi$ is normalized such that (66) is valid, then (9) is also the marginal probability that the particle will be found at $t$. Of course, in practice a measurement allways lasts a finite time $\Delta t$ and the detection time $t$ cannot be determined with perfect accuracy. Thus, (8) should be viewed as a limiting case in which the fundamental probability ([6) is averaged over a very small $\Delta t$. More precisely, if the particle is detected between $t-\Delta t / 2$ and $t+\Delta t / 2$, then (8) is the probability of different 3 -space positions of the particle detected during this small $\Delta t$.

Can the probabilistic interpretation (6) be verified experimentally? In fact, it already is! In practice one often measures cross sections associated with scaterring experiments or decay widths and lifetimes associated with spontaneous decays of unstable quantum systems. These experiments agree with standard theoretical predictions. Our point is that these standard theoretical predictions actually use (6) , although not explicitly. Let us briefly explain it. (A complete explanation requires an explicit and careful account of all normalization factors. Since this is not essential for understanding the basic idea, 
a complete analysis will be presented elsewhere.) The basic theoretical tool in these predictions is the transition amplitude $A$. Essentially, the transition amplitude is the wave function (usually Fourier transformed to the 3-momentum space) at $t \rightarrow \infty$, calculated by assuming that the wave function at $t \rightarrow-\infty$ is known. Due to energy conservation one obtains $A \propto \delta\left(E_{\text {in }}-E_{\text {fin }}\right)$, where $E_{\text {in }}$ and $E_{\text {fin }}$ are the initial and final energy, respectively. Thus, the transition probability is proportional to $|A|^{2} \propto\left[\delta\left(E_{\text {in }}-E_{\text {fin }}\right)\right]^{2}=(T / 2 \pi) \delta\left(E_{\text {in }}-\right.$ $\left.E_{\text {fin }}\right)$, where $T=\int d t=2 \pi \delta(E=0)$. Since $T$ is infinite, this transition probability is physically meaningless. The standard interpretation (see, e.g., [8] for the nonrelativistic case or [9] for the relativistic case), which agrees with experiments, is that the physical quantity is $|A|^{2} / T$ and that this quantity is (proportional to) the transition probability per unit time. But this is essentially the same as our equation (6) which says that $\int d^{3} x|\psi|^{2}$ is not probability itself, but probability per unit time. Although the interpretation of $|A|^{2} / T$ as probability per unit time may seem plausible even without explicitly postulating (6) , without this postulate such an interpretation of $|A|^{2} / T$ is at best heuristic and cannot be strictly derived from other basic postulates of QM, including (7). In this sense, the standard interpretation of transition amplitudes in terms of transition probabilities per unit time is better founded in basic axioms of $Q M$ if (6) is also adopted as one of its axioms.

\section{Dynamics and generalization to many particles}

So far we have been explicitly discussing only the kinematics. Now let us include the dynamics. For simplicity, we shall only consider particles without spin. Thus, the wave function of a free relativistic particle satisfies the Klein-Gordon equation

$$
\left[\hat{p}^{\mu} \hat{p}_{\mu}-m^{2}\right] \psi(x)=0
$$

The interaction with a background field $A^{\mu}(x)$ can also be included through the substitution $\hat{p}^{\mu} \rightarrow \hat{p}^{\mu}+e A^{\mu}(x)$, but the interaction will not influence our conclusions, so we consider only the free case. By writing $\psi(x)=e^{-i m t} \psi_{\mathrm{NR}}(\mathbf{x}, t)$ and taking the nonrelativistic limit of (10), one finds that $\psi_{\mathrm{NR}}$ satisfies the nonrelativistic Schrödinger equation. Hoping that it will not cause confusion, in the rest of the discussion we omit the label "NR" in $\psi_{\mathrm{NR}}$, so the nonrelativistic Schrödinger equation can be written as

$$
\hat{H} \psi(\mathbf{x}, t)=\hat{E} \psi(\mathbf{x}, t)
$$

where $\hat{H}=\hat{\mathbf{p}}^{2} / 2 m$ is the dynamical Hamiltonian operator that acts in the space of functions of $\mathbf{x}$, while $\hat{E}$ given by (4) is the kinematic energy operator that acts in the space of functions of $t$. Since $\hat{H}$ is positive definite, Eq. (11) implies that wave functions of the form $e^{-i E t} \chi(\mathbf{x})$ may be solutions only if $E \geq 0$. This means that the $\delta$-function

$$
\delta(t)=\int_{-\infty}^{\infty} \frac{d E}{2 \pi} e^{-i E t}
$$

cannot be constructed from solutions of (11). In other words, in the nonrelativistic case eigenstates of the time operator are not physical. 
Can this problem be avoided by using relativistic QM? Eq. (10) contains solutions with both positive and negative energies $E$. Nevertheless, the spectrum of allowed energies is restricted again, by the condition $|E| \geq m$. Moreover, even the case $m=0$ is problematic, because it is usually assumed that (for uncharged particles) only positive energy solutions of (10) are physical. (There are many ways to explain why only positive E's are physical. One way is to observe that only with that restriction Eq. (10) can be reduced to Eq. (11) in the nonrelativistic limit. Another way is to use relativistic quantum field theory, where physical states are obtained by acting with creation operators $\hat{a}^{\dagger}(\mathbf{p})$ on the vacuum, which create states with positive $E$ only [10].)

Thus, we see that eigenstates of the time operator cannot be constructed from physical solutions of the dynamical equations of motion. Does it mean that our kinematic time operator is physically inconsistent? For example, the time operator cannot be represented in terms of physical states as $\hat{t}=\int d t|t\rangle t\langle t|$. Nevertheless, this fact by itself does not yet represent a physical problem, as long as this operator still exists kinematically. Still, a physical problem would occur if this would imply that time cannot be measured. But this leads us to a much more controversial aspect of QM - the theory of measurement.

Before discussing the problems related to quantum measurements, let us first clarify some additional, less problematic, aspects of our approach. Another problem with physical solutions of (10) and (11) is that they cannot be localized in time, i.e., they satisfy $\int_{-\infty}^{\infty} d t|\psi|^{2}=\infty$. Strictly speaking, this means that $\psi$ cannot be normalized such that $\langle\psi \mid \psi\rangle=1$. Nevertheless, we do not see this as a real physical problem. After all, no experiment lasts an infinite time. And even if it does, one can allways introduce a large but finite time-cutoff $T$ such that $\int_{-T / 2}^{T / 2} d t|\psi|^{2}$ is finite, and put $T \rightarrow \infty$ at the end of calculation. Indeed, as we already discussed above, a regularization of that kind is a standard procedure in calculations of transition probabilities per unit time and no serious problems have been found so far.

Another aspect that we want to discuss briefly is the generalization to wave functions of $n$ particles. As we already pointed out, the main idea is to treat time on an equal footing with space. Thus, each particle has its own space position $\mathbf{x}_{a}, a=1, \ldots, n$, as well as its own time coordinate $t_{a}$. Therefore, the wave function is of the form $\psi\left(x_{1}, \ldots, x_{n}\right)$, which is a many-time wave function [11]. Then (6) generalizes to

$$
d P=\left|\psi\left(x_{1}, \ldots, x_{n}\right)\right|^{2} d^{4} x_{1} \cdots d^{4} x_{n} .
$$

Hence, if the first particle is detected at $t_{1}$, second particle at $t_{2}$, etc., then Eq. (8) generalizes to

$$
d P_{(3 n)}=\frac{\left|\psi\left(\mathbf{x}_{1}, t_{1}, \ldots, \mathbf{x}_{n}, t_{n}\right)\right|^{2} d^{3} x_{1} \cdots d^{3} x_{n}}{N_{t_{1}, \ldots, t_{n}}}
$$

where

$$
N_{t_{1}, \ldots, t_{n}}=\int\left|\psi\left(\mathbf{x}_{1}, t_{1}, \ldots, \mathbf{x}_{n}, t_{n}\right)\right|^{2} d^{3} x_{1} \cdots d^{3} x_{n} .
$$

If these particles do not interact, then (in the relativistic case) $\psi$ satisfies $n$ Klein-Gordon equations $\left[\hat{p}_{a}^{\mu} \hat{p}_{a \mu}-m_{a}^{2}\right] \psi=0$, one for each $a$. They can also be summed to give a single $n$-particle Klein-Gordon equation

$$
\sum_{a}\left[\hat{p}_{a}^{\mu} \hat{p}_{a \mu}-m_{a}^{2}\right] \psi\left(x_{1}, \ldots, x_{n}\right)=0
$$


By taking the nonrelativistic limit and considering the coincident case $t_{1}=\cdots=t_{n} \equiv t$, one finds that these equations reduce to the standard single-time nonrelativistic equations of $n$-particle systems.

\section{Quantum measurements and the Bohmian inter- pretation}

Now let us discuss our final and the most difficult issue - the issue of quantum measurements. According to the standard interpretation of QM, when a physical observable is measured, then the wave function collapses to an eigenstate of the operator describing this observable. However, as we have seen, eigenstates of the time operator are unphysical. At first sight it may seem to be a serious drawback of our approach. Nevertheless, we argue that it is actually a virtue, rather than a drawback. Namely, even though such a time operator is not consistent within the standard interpretation of QM, it may still be consistent within some of the alternative interpretations. Usually, the question of "correct" interpretation is viewed as something that belongs to philosophy, rather than science. But now we propose a new scientific criterion for distinguishing between acceptable and unacceptable interpretations; in an acceptable interpretation the fact that time eigenstates are not physical should not imply that time itself is unmeasurable.

It is beyond the scope of this paper to discuss all acceptable interpretations. Instead, we concentrate on one such interpretation - the Bohmian one [12]. According to this interpretation, in experiments we actually do not detect wave functions, but pointlike particles that move deterministically through spacetime and exist as objects separated from (although guided by) the wave function. This explains why the spacetime position of a particle makes physical sense even without eigenstates of the time operator (see also [13]). But how can time be measured? According to the Bohmian interpretation, all quantum measurements eventually reduce to measurements of 3 -space positions of particles of the measuring apparatuses [12], which then also applies to the measurement of "time" by a real clock. (For explicit models of a clock see, e.g., [14.)

The Bohmian interpretation has also other advantages (see, e.g., [15]). Moreover, many objections against this interpretation have been found to be unjustified. In particular, contrary to frequent claims, it was found that the Bohmian interpretation does have a practical use [16], that the Bohmian particle velocities are measurable [17] (in the sense of weak measurements [18]), and that (an improved version of) Bohmian mechanics can describe particle creation and destruction, by using either quantum field theory [19] or string theory [20, 21]. In addition, it is sometimes objected that the Bohmian interpretation is nonlocal, but this is not really a valid argument against this particular interpretation because any theory (compatible with QM) that assumes that single reality exists even without measurements must necessarily be nonlocal [22].

Finally, due to nonlocality, it is frequently objected that this interpretation is not consistent with relativity. Nevertheless, various partial steps towards a relativistic-covariant formulation of the Bohmian interpretation of many-particle systems have been done in [23] and [20]. Here we make a synthesis of these partial steps with the results of the present paper to show in a simple and concise way that the Bohmian interpretation is 
indeed relativistic covariant, despite of being nonlocal.

Each Bohmian particle has a trajectory $X_{a}^{\mu}(s)$, where $s$ is an auxiliary scalar parameter along the trajectories. Since $\psi\left(x_{1}, \cdots, x_{n}\right)$ does not depend on $s$, by writing $\psi=|\psi| e^{i S}$ one finds that (16) implies a relativistic conservation equation

$$
\frac{\partial|\psi|^{2}}{\partial s}+\sum_{a} \partial_{a \mu}\left(|\psi|^{2} v_{a}^{\mu}\right)=0
$$

where

$$
v_{a}^{\mu}\left(x_{1}, \cdots, x_{n}\right)=-\partial_{a}^{\mu} S\left(x_{1}, \cdots, x_{n}\right) .
$$

Therefore, it is consistent to postulate that the trajectories satisfy

$$
\frac{d X_{a}^{\mu}(s)}{d s}=v_{a}^{\mu}\left(X_{1}(s), \cdots, X_{n}(s)\right)
$$

Namely, if an ensemble of particles has the distribution (13) for some initial $s$, then (17) and (19) guarantee that it will have the same distribution for any $s$. The Bohmian equation of motion (19) is nonlocal because it says that the velocity of a particle for some value of $s$ depends on the positions of all other particles for the same value of $s$. Nevertheless, it is clear that this equation is manifestly relativistic covariant. In fact, the auxiliary parameter $s$ can be completely eliminated and (19) can be rewritten as an equation that determines particle trajectories in spacetime without any referring to the parameter $s$ [23]. Finally, we also note that the velocities (19) can be even measured in the sense of weak measurements, completely analogously to the weak measurements of nonrelativistic Bohmian velocities [17]. To conclude, all this shows that the Bohmian interpretation is well motivated, relativistic covariant, and compatible with the relativistic invariant probabilistic interpretation (13).

\section{Acknowledgements}

This work was supported by the Ministry of Science of the Republic of Croatia under Contract No. 098-0982930-2864.

\section{References}

[1] P. Busch, quant-ph/0105049.

[2] K. Boström, quant-ph/0301049.

[3] H. Nikolić, Found. Phys. 37, 1563 (2007).

[4] W. Pauli, Handbuch der Physik (Springer, Berlin, 1926).

[5] E. C. G. Stückelberg, Helv. Phys. Acta 14, 322 (1941); Helv. Phys. Acta 14, 588 (1941); L. P. Horwitz and C. Piron, Helv. Phys. Acta 46, 316 (1973); A. Kyprianidis, Phys. Rep. 155, 1 (1987); J. R. Fanchi, Found. Phys. 23, 487 (1993). 
[6] D. Marolf and C. Rovelli, Phys. Rev. D 66, 023510 (2002).

[7] T. D. Newton and E. P. Wigner, Rev. Mod. Phys. 21, 400 (1949).

[8] L. I. Schiff, Quantum Mechanics (McGraw-Hill, Singapore, 1968).

[9] F. Halzen and A. D. Martin, Quarks and Leptons (John Willey \& Sons, New York, 1984); J. D. Bjorken and S. D. Drell, Relativistic Quantum Mechanics (McGraw-Hill, New York, 1964).

[10] L. H. Ryder, Quantum Field Theory (Cambridge University Press, Cambridge, 1984).

[11] S. Tomonaga, Prog. Theor. Phys. 1, 27 (1946); P. Ghose and D. Home, Phys. Rev. A 43, 6382 (1991).

[12] D. Bohm, Phys. Rev. 85, 166 (1952); Phys. Rev. 85, 180 (1952); D. Bohm and B. J. Hiley, Phys. Rep. 144, 323 (1987); P. R. Holland, The Quantum Theory of Motion (Cambridge University Press, Cambridge, 1993).

[13] C. R. Leavens, Phys. Lett. A 178, 27 (1993).

[14] H. Salecker and E. P. Wigner, Phys. Rev. 109, 571 (1958); J. R. Fanchi, Phys. Rev. A 34, 1677 (1986).

[15] D. Bohm and B. J. Hiley, Phys. Rev. Lett. 55, 2511 (1985); D. Bohm, C. Dewdney, and B. J. Hiley, Nature 315, 294 (1985); C. Dewdney, P. R. Holland, A. Kyprianidis, and J. P. Vigier, Nature 336, 536 (1988).

[16] C. L. Lopreore and R. E. Wyatt, Phys. Rev. Lett. 82, 5190 (1999).

[17] H. M. Wiseman, New J. Phys. 9, 165 (2007); D. Dürr, S. Goldstein, and N. Zanghì, arXiv:0808.3324.

[18] Y. Aharonov, D. Z. Albert, and L. Vaidman, Phys. Rev. Lett. 60, 1351 (1988).

[19] D. Dürr, S. Goldstein, R. Tumulka, and N. Zanghì, Phys. Rev. Lett. 93, 090402 (2004); H. Nikolić, Found. Phys. Lett. 17, 363 (2004); H. Nikolić, Found. Phys. Lett. 18, 123 (2005).

[20] H. Nikolić, hep-th/0702060 (v3).

[21] H. Nikolić, arXiv:0705.3542, to appear in Europhys. Lett.

[22] J. S. Bell, Speakable and Unspeakable in Quantum Mechanics (Cambridge University Press, Cambridge, 1987).

[23] K. Berndl, D. Dürr, S. Goldstein, and N. Zanghì, Phys. Rev. A 53, 2062 (1996); H. Nikolić, Found. Phys. Lett. 18, 549 (2005); H. Nikolić, AIP Conf. Proc. 844, 272 (2006); quant-ph/0512065. 